\title{
Effect of short-term variation in irradiance on light harvesting and photosynthesis of the marine diatom Skeletonema costatum: a laboratory study simulating vertical mixing
}

\author{
JaCco KromkamP* and Marga LimbeeK \\ Netherlands Institute of Ecology, Centre for Estuarine and Coastal Ecology, Vierstraat 28, NL-440I EA Yerseke, \\ The Netherlands
}

(Received 7 September 1992; revised 15 April 1993; accepted 27 April 1993)

\begin{abstract}
A laboratory study was conducted into the physiology of Skeletonema costatum grown under a simple sinusoidal and a fluctuating light regime. The latter simulated a light regime similar to that which could result from the vertical mixing caused by Langmuir circulation. It was shown that the culture simulating vertical mixing reacted by decreasing the photosynthetic unit (PSU) size and increasing the number of PSUs, and hence optimized the rate of maximal photosynthesis at high, saturating irradiances. This culture also showed some change in photosynthetic parameters during the light period, which was especially pronounced during the shift from a low to a higher irradiance. The effect of this on estimates of primary production in a water column is discussed. Further, it is speculated that the assimilation number is regulated by the maximum light intensity experienced during the day rather than the total daily light dose, because only the culture submitted to a fluctuating light regime showed a real change in the maximum rate of photosynthesis $\left(P_{\max }^{\mathrm{B}}\right)$ upon transfer to higher light levels.
\end{abstract}

\section{Introduction}

When primary production is estimated, the photosynthesis versus irradiance curve forms the basis for the calculation of phytoplankton production. The implicit assumptions made in this calculation are that the functional relationship between light and photosynthesis does not change in time when the algae are circulating through a light gradient in a mixed layer and that this relationship stays constant during the period of the measurement. Whether these assumptions are valid depends on the time scale of fluctuation in the physical environment and the time scale of change of the photosynthetic response. It is generally assumed that when the time scale of change is larger than the time scale of adaptation, phytoplankton will conform to a vertical gradient associated with the adaptation process (Reynolds et al., 1987; Cullen \& Lewis, 1988).

*Author for correspondence. Tel. 1131 1920, fax 11313136 , email surf230@kub.nl.

\footnotetext{
Abbreviations: $k_{\mathrm{c}}$, chlorophyll-specific absorption cross-section; $\sigma$, optical cross-section; PSU, photosynthetic unit; $\tau$, overall photosynthetic turnover time; $1 / \Phi_{\vec{p}}$, minimum quantum requirement; $P_{\max }^{\mathrm{B}}$, maximum rate of photosynthesis; $\alpha^{\mathrm{B}}$, photosynthetic efficiency; HL, high light; LL, low light; TDLD, total daily light dose.
}

It is well known that phytoplankton respond physiologically to changes in the ambient light field. For reviews on light-shade adaptation, see Harris (1978), Richardson et al. (1983), Falkowski (1980). However, changes in the nutrient regime (Riegman et al., 1985; Kromkamp et al., 1989; Herzig \& Falkowski, 1989; Kolber et al., 1988) as well as changes in the ambient light regime can cause alterations in photosynthetic parameters. We now have a reasonable understanding of the mechanisms causing changes in photosynthetic parameters from studies performed on photoadaptation. The problem is, however, that the best-documented studies were performed on algal cultures adapted to a particular set of environmental conditions. The major causes for the short-term variations in photosynthetic parameters are less well known, despite the numerous studies on short-term variations in irradiance (Marra, 1978; Neale \& Marra, 1985). Nevertheless, a few studies report on the kinetics of photoadaptation (Post et al., 1984, 1985; Post, 1986; Cullen \& Lewis, 1988) and it is clear from them that photoadaptation normally follows first order kinetics or, occasionally, a logistic function (Cullen \& Lewis, 1988).

A number of questions remain. For example, in lightlimited cultures, it is still unknown whether the increase in photosynthetic capacity observed when the growth 
irradiance is reduced is due to a decreased light intensity or a lower total daily light dose causing a lower growth rate. This is because it is very difficult, in light-limited cultures, to separate growth effects from direct irradiance effects.

In this paper we describe the photosynthesis of lightlimited continuous cultures of the marine diatom Skeletonema costatum, grown with a simple, sinusoidal light regime and with a fluctuating light regime simulating vertical mixing, both grown at identical dilution rate (i.e. equal growth rates). However, the total amount of light quanta received during the $24 \mathrm{~h}$ light-dark period was the same. This made it possible to separate the long-term from the short-term light effects. It will be shown that despite similar light doses, photosynthetic physiology was quite different.

\section{Methods}

Organism and cultivation. S. costatum was isolated by J. Rijstenbil from the 'Veerse Meer', part of the Dutch Delta area. The organism was grown in a modified artificial medium with a salinity of approximately $14 \mathrm{~g}^{-1}$, according to Kester et al. (1967), which consisted of the following salts: $\mathrm{NaCl}, 327 \mathrm{~mm} ; \mathrm{Na}_{2} \mathrm{SO}_{4}, 23 \mathrm{~mm}$; $\mathrm{MgCl}_{2}, 43 \mathrm{~mm}$; KCl, $7.2 \mathrm{~mm} ; \mathrm{CaCl}_{2}, 8.3 \mathrm{~mm} ; \mathrm{KBr}, 330 \mu \mathrm{M} ; \mathrm{NaNO}_{3}$, $5.9 \mathrm{~mm} ; \mathrm{H}_{3} \mathrm{BO}_{3}, 178 \mu \mathrm{M} ; \mathrm{NaH}_{2} \mathrm{PO}_{4}, 50 \mu \mathrm{M} ; \mathrm{NaHCO}_{3}, 2 \mathrm{mM} ; \mathrm{SrCl}_{2}$, $38 \mu \mathrm{m} ; \mathrm{Na}_{2} \mathrm{SiO}_{3}, 150 \mu \mathrm{M} ; \mathrm{Na}_{2} \mathrm{EDTA}, 6.3 \mu \mathrm{m} ; \mathrm{FeCl}_{2}, 10 \mu \mathrm{m}$. Trace metals: $\mathrm{CuSO}_{4}, 6 \mathrm{~nm} ; \mathrm{Na}_{2}$ EDTA, $310 \mathrm{~nm} ; \mathrm{ZnSO}_{4}, 81 \mathrm{~nm} ; \mathrm{MnCl}_{2}$, $198 \mathrm{~nm} ; \mathrm{CoCl}_{2}, 19 \mathrm{nM} ; \mathrm{Na}_{2} \mathrm{MoO}_{4}, 56 \mathrm{nM}, \mathrm{Na}_{2} \mathrm{SeO}_{3}, 6 \mu \mathrm{M}$. Vitamins: thiamine (B1), $74 \mathrm{nM}$; biotine (H), $100 \mathrm{nM}$; cyanocobalamin (B12), $18 \mathrm{~nm}$. The medium was sparged with air and after the $\mathrm{pH}$ was adjusted to 7.8 the medium was filter-sterilized (IVEX-2, $0.22 \mu \mathrm{m}$ pore size, Millipore) by pumping it into autoclaved polycarbonate carboys (Nalgene).

The algae were cultured in flat, 21 cyclindrical glass vessels with a light path length of $3 \mathrm{~cm}$. In front of the vessel a water jacket was mounted connected to a temperature-controlled water bath to keep growth at $17^{\circ} \mathrm{C}$. The algae remained suspended by gently bubbling the cultures with water-saturated air. Nevertheless, sometimes limited cell lysis $(<5 \%)$ could not be prevented.

The light course was a $250 \mathrm{~W}$ (culture 1) or $400 \mathrm{~W}$ (culture 2, see below) high pressure lamp (Philips HPIT E40). The cultures were placed in a black box. In front of the culture (facing the lamp) a system of horizontal slats (Venetian blinds) was mounted. The position of the slats was altered by a step motor, controlled by a computer. Three serially aligned custom-built silicon light sensors were attached to the back or the front of the culture vessel. These sensors had an identical linear response over the range of irradiances used. The actual irradiance measured by the sensors was compared with the calculated irradiance (see below) and when necessary the position of the slats was adjusted (every 5 s). In this way the actual irradiance was always within $5 \%$ of the calculated value.

The average irradiance $(I)$ in the culture was calculated as follows (Van Liere \& Walsby, 1982):

$$
I=\left(I_{0}-I_{\mathrm{d}}\right) /\left[\ln \left(I_{0}\right)-\ln \left(I_{\mathrm{d}}\right)\right]
$$

where $I_{0}$ and $I_{\mathrm{d}}$ are the irradiance at the back of the culture or of a Dubinsky chamber (Dubinsky et al., 1987) filled with water or with algae, respectively.

Culture 1 received a sinusoidal light regime, simulating the surface irradiance $\left(I_{s}\right)$ on a day with a clear sky (Kirk, 1983):

$$
I_{\mathrm{s}}=I_{\mathrm{m}} \sin (\pi t / \mathrm{LP})
$$

where $I_{\mathrm{m}}$ is the maximum irradiance at noon, $t$ the time since sunrise (i.e. start light period) and LP is the length of the light period in a $24 \mathrm{~h}$ light-dark (LD) cycle.

Culture 2 simulated vertical mixing, i.e. it was assumed that the average movement through the mixed layer to the depth could be described by a circular motion: this means that irradiance at a hypothetical vertical position can be described as a sine function of time and the number of cycles (nc) during the above-mentioned light period from the surface to the bottom of the mixed layer $\left(z_{\mathrm{m}}\right)$ :

$$
z_{\mathrm{t}}=0 \cdot 5 z_{\mathrm{m}}[1+\sin (2 \pi \mathrm{nct} / \mathrm{LP}-0.5 \pi)]
$$

where $z_{\mathrm{t}}$ is the depth $(\mathrm{m})$ at time $t$. The irradiance at depth $z$ is calculated using the Beer-Lambert equation:

$$
I_{\mathrm{z}}=I_{\mathrm{s}} \mathrm{e}^{-K_{\mathrm{d}} z}
$$

where $K_{d}$ is the diffuse downwelling attenuation coefficient $\left(\mathrm{m}^{-1}\right)$. Of course, particle displacement by diffuse turbulent motion is more complicated than addressed here, but circular movement through the mixed layer can be caused by, for instance, Langmuir circulation (although mixing times are in general shorter then, see below). The use of equations 2, 3 and 4 makes it possible to simulate all sorts of light gradients in the mixed layer. During the first experiment both cultures were grown with a light period of $8 \mathrm{~h}$ and an average low light intensity (LL) of $30 \mu \mathrm{E} \mathrm{m}^{-2} \mathrm{~s}^{-1}$ [i.e. a total daily light dose (TDLD)] of $0.86 \mathrm{E} \mathrm{m}^{-2}$. The peak irradiance $\left(I_{\mathrm{m}}\right)$ of culture 1 was $47 \mu \mathrm{E} \mathrm{m}^{-2} \mathrm{~s}^{-1}$. Algae in culture 2, simulating vertical mixing, were assumed to move three times from the surface to $z_{\mathrm{rr}}$ and back during the $8 \mathrm{~h}$ light period. Hence, the average time to traverse the mixed layer once from the surface to the bottom of the mixed layer was $80 \mathrm{~min}$. Using the approach of Denman \& Gargett (1983) one can calculate that this equals the mixing time of a mixed layer of $30.40 \mathrm{~m}$ depth with an average turbulent velocity generated by mild windspeeds $\left(2-3 \mathrm{~m} \mathrm{~s}^{-1}\right)$. The maximal irradiance in this culture was $167 \mu \mathrm{E} \mathrm{m}^{-2} \mathrm{~s}^{-1}$ (this higher peak irradiance was necessary to get similar TDLDs). The time course in average irradiance of both cultures is depicted in Fig. 1. After the steady states were measured, the light intensity was in one step doubled to a TDLD of $1.72 \mathrm{E} \mathrm{m}^{-2}$ (referred to as high light (HL)], giving maximal irradiances of 100 and $320 \mu \mathrm{E} \mathrm{m}^{-2} \mathrm{~s}^{-1}$. During steady state the dilution rate (which equals growth rate in steady state) at LL was set at $0.07 \mathrm{~d}^{-1}$ and for $\mathrm{HL}$ at $0 \cdot 18 \mathrm{~d}^{-1}$.

Optical measurements. Irradiances were measured with a cosinecorrected quantum sensor (MACAM SD101Q). This light meter was

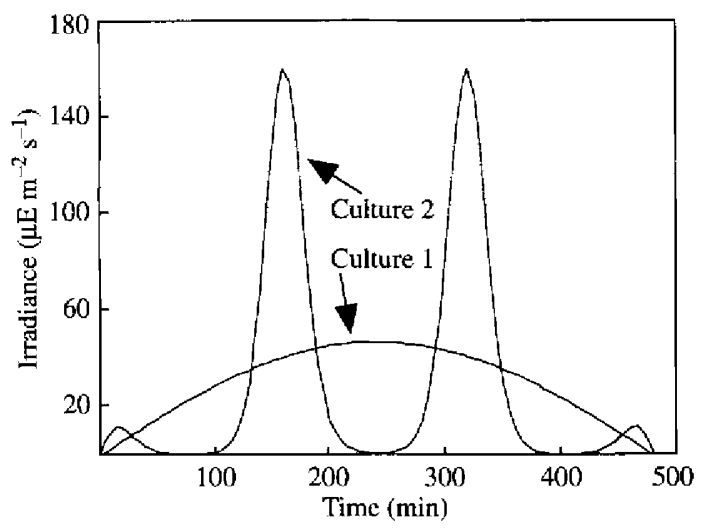

Fig. 1. Change in light intensity in cultures 1 and 2 during growth in LL. In HL the intensities were doubled. 
also used to calibrate the silicon light sensors used in the continuous cultures.

In vivo absorption of the algal cells was measured in a double-beam scanning spectrophotometer (Uvikon 940) according to the Shibata technique (Shibata et al., 1954) to minimize the loss of signal due to scattering: the cuvettes were close $(1.5 \mathrm{~cm})$ to the windows of the photomultiplier (PMT) and a light diffusor was placed between the PMT and the cuvette. Chlorophyll-specific absorption cross-sections $\left.\left[k_{\mathrm{c}}, \mathrm{m}^{2} \text { (mg chl }\right)^{-1}\right]$ were calculated from the absorption measurements and the chlorophyll concentration:

$$
k_{\mathrm{c}}=[A \times 100 \times \ln (10)] /[\text { chlorophyll }]
$$

where $A$ is the in vivo absorption of the samples.

The optical cross-section of a single chlorophyll $a$ molecule $\left(\sigma_{\mathrm{chl}}, \mathrm{nm}^{2}\right)$ can be calculated from the absorption cross-section $k_{\mathrm{c}}$ by changing the dimensions from $\mathrm{m}^{2}(\mathrm{mg} \mathrm{chl})^{-1}$ to $\mathrm{nm}^{2}$ (molecule chl) $)^{-1}$.

Photosynthesis measurements. Photosynthetic oxygen evolution was measured in a chamber using a polarographic oxygen electrode (YSI 5331) fitted with a water jacket to keep the temperature constant $\left(17 \pm 0.01{ }^{\circ} \mathrm{C}\right)$ (Dubinsky et al., 1987). A slide projector with neutral density filters was used as a light source for photosynthesis measurements. Respiration was measured before the light measurements were done. Light was measured at the back of the chamber. The average irradiance in the chamber was measured as described by equation 1 . Photosynthetic irradiance data, normalized to chlorophyll $a$, were fitted with an iterative least-squares method according to Eilers \& Peeters (1988). The maximal photosynthetic capacity $\left(P_{\max }^{\mathbf{B}}\right)$ is the maximum rate of photosynthesis $\left[\mathrm{mg} \mathrm{O}_{2}(\mathrm{mg} \mathrm{chl})^{-1} \mathrm{~h}^{-1}\right.$ ]. Gross $P_{\max }^{\mathrm{B}}$ values are presented here. The photosynthetic efficiency is the slope $\left(\alpha^{\mathrm{B}}\right)$ of the photosynthesis versus irradiance curve $\left[\mathrm{mg} \mathrm{O}_{2}(\mathrm{mg} \mathrm{chl})^{-1} \mathrm{~h}^{-1}\right)$ $\left.\left(\mu \mathrm{E} \mathrm{m}^{-2} \mathrm{~s}^{-1}\right)^{-1}\right]$. The superscript $\mathrm{B}$ in $\alpha^{\mathrm{B}}$ and $P_{\max }^{\mathrm{B}}$ denotes that the photosynthetic parameters are expressed per unit chlorophyll $a$.

The photosythetic unit (PSU) size, i.e. the number of chlorophyll $a$ molecules necessary to produce one molecule of $\mathrm{O}_{2}$, was determined by exposing a sample of known chlorophyll a concentration to a series of single turnover saturating flashes of different frequency (Myers \& Graham, 1971; Dubinsky et al., 1986). A PSU is thus an oxygenproducing functional entity. The rate of oxygen production (being a linear function of flash frequency) was measured in the chamber described above. Because the flash unit (EG\&G MVS 2601) was equipped with a storage capacitor, the energy of each xenon flash was constant. Flash duration was less than $10 \mu$ s, i.e. less than the turnover time of the $Q_{A}$ and thus short enough to close photosynthetic reaction centres only once. The intensity of the flash was high enough to close all photosynthetic reaction centres. No background photosystem 1 (PSI) light (far red light) was provided because in diatoms the optical cross-section of PSI for xenon light is large enough (i.e. PSI-activity will not be rate-limiting, Dubinsky $e^{t}$ al., 1986). When the rate of oxygen evolution $\left[\mathrm{mol} \mathrm{O}_{2}(\mathrm{~mol} \mathrm{chl})^{-1} \mathrm{~s}^{-1}\right]$ is plotted against the flash frequency $\left(\mathrm{s}^{-1}\right)$, the reciprocal of the slope of the graph is equal to the PSU size $\left[\mathrm{mol} \mathrm{chl}\left(\mathrm{mol} \mathrm{O}_{2}\right)^{-1}\right]$. The slope was calculated using linear regression analyses. As four electrons have to be photoactivated to produce one molecule of oxygen, each PSU contains four PSII reaction centres.

The overall turnover time $(\tau, \mathrm{ms}$, i.e. the time necessary to move four electrons through one PSU and donate them to NADP) determines the rate of photosynthesis. $\tau$ was calculated from the PSU size and $P_{\max }^{\mathrm{B}}$ (expressed per minute) (Dubinsky et al., 1986):

$$
\tau=60000 /\left(\mathrm{PSU} \times P_{\max }^{\mathrm{B}}\right)
$$

The factor 60000 is a conversion factor $\left(\mathrm{ms} \mathrm{min}^{-1}\right)$.

The optical cross-section of a PSU $\left(\sigma_{\mathrm{PSC}}, \mathrm{nm}^{2}\right)$ was calculated as (Dubinsky et al., 1986):

$$
\sigma_{\mathrm{PSU}}=\sigma_{\mathrm{chl}} \times \mathrm{PSU}
$$

The minimum quantum requirement [mol quanta $\left.\left(\mathrm{mol} \mathrm{O}_{3}\right)^{-1}\right]$ is the reciprocal of the quantum efficiency of photosynthesis $\left(\Phi_{\mathrm{p}}\right)$ :

$$
1 / \Phi_{\mathrm{P}}=115 \times k_{\mathrm{c}} / \alpha^{\mathrm{B}}
$$

where the constant 115 is needed to unify the dimensions of $k_{\mathrm{c}}\left[\mathrm{m}^{2}\left(\mathrm{mg} \mathrm{chl}^{-1}\right]\right.$ and $\alpha^{\mathrm{B}}\left[\mathrm{mg} \mathrm{O}_{2}(\mathrm{mg} \mathrm{chl})^{-1} \mathrm{~h}^{-1}\left(\mu \mathrm{E} \mathrm{m}^{-2} \mathrm{~s}^{-1}\right)^{-1}\right]$.

Chemical analysis. Pigments were measured in 90\% (v/v) acetone and measured spectrophotometrically using the equations of Jeffrey \& Humphrey (1975). Samples $(4 \mathrm{ml})$ were spun down and sonicated three times for $10 \mathrm{~s}$ on ice in $10 \mathrm{ml} 90 \%$ acetone. After adding another $6 \mathrm{ml}$ of $90 \%$ acetone, the samples were put in the dark, at room temperatuie, for extraction. After spinning $(900 \mathrm{~g}, 5 \mathrm{~min})$ the supernatant was scanned. No chlorophyll could be extracted from the pellet.

Transient states. During the transient state from LL to HL the data were fitted to a first order kinetic equation (Falkowski, 1980; Post $e t$ al., 1984):

$$
P_{1}=\left(P_{0}-P_{\infty}\right) \mathrm{e}^{-k t}+P_{\infty}
$$

where $P_{\mathrm{t}}$ is the process parameter during the transient state at time $t . P_{0}$ and $P_{\infty}$ are the initial and final values for parameter $P$, and $k$ is the specific rate of adaptation having dimensions of $\mathrm{h}^{-1} . P_{0}, P_{\infty}$ and $k$ were estimated by non-linear least-squares regression using the software program SYSTAT for Windows (Version 5.0, Systat Inc. 1992, Evanstone, IL, USA).

\section{Results and Discussion}

\section{In vivo absorption cross-section}

Cells of the example shown (Fig. 2) grown under LL had different average $k_{c}$ values of 0.0090 and $0.0073 \mathrm{~m}^{2}$ $(\mathrm{mg} \mathrm{chl})^{-1}$ for the cultures receiving a sinusoidal (1) and fluctuating (2) light regime, respectively. Since $k_{\mathrm{c}}$ was identical at $675 \mathrm{~nm}$, the small differences in absorption cross-section cannot be due to differences in self-shading of chlorophyll by the thylakoids within the chloroplasts, but were more likely due to a small difference in pigment composition. The $k_{\mathrm{c}}$ of both cultures was measured several times at different days during the steady state. The average values ( $\pm 95 \%$ confidence intervals) were

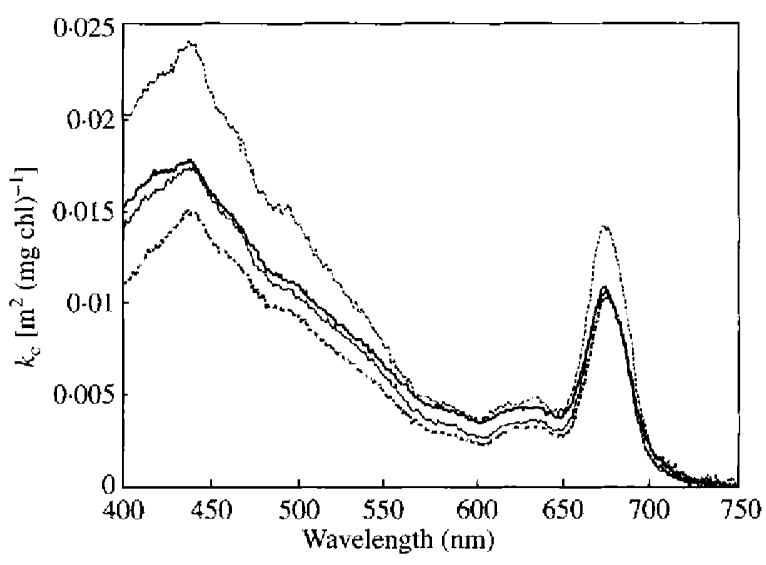

Fig. 2. Absorption cross-section ( $\left.k_{c}\right)$ of cells grown in LL (bold lines) or HL (thin lines). Solid lines, culture 1; dashed lines, culture 2. 


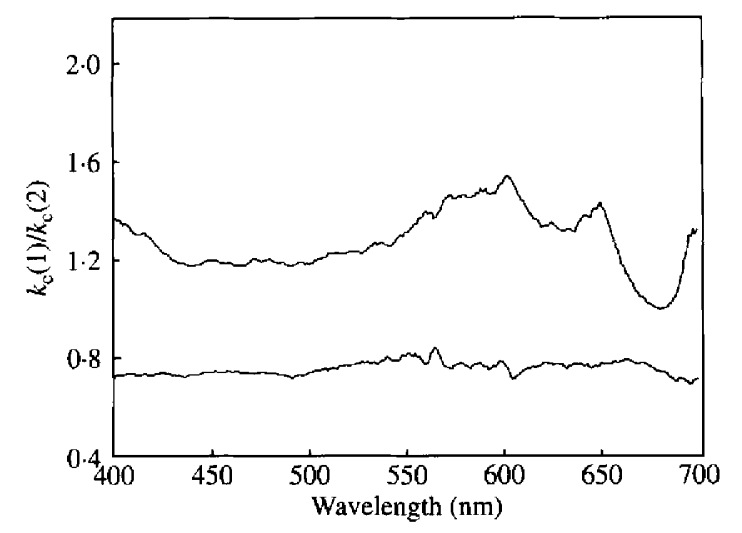

Fig. 3. Relative changes in $k_{\mathrm{c}}$ determined as the quotient of the $k_{\mathrm{c}}$ values from cultures 1 and 2 grown in LL (top line) and HL (lower line).

$0.0087( \pm 0.003)$ and $0.0080( \pm 0.0006) \mathrm{m}^{2}(\mathrm{mg} \mathrm{chl})^{-1}$ for cultures 1 and 2 , respectively, hence they were not significantly different. Our $k_{\mathrm{c}}$ values were similar to those reported for $S$. costatum by Welschmeyer \& Lorenzen (1981), but were higher than the 0.0038-0.0044 $\mathrm{m}^{2}(\mathrm{mg}$ (chl) ${ }^{-1}$ reported for the marine diatom Thalassiosira weisflogii (Dubinsky et al., 1986; Post et al., 1985).

When the cells were grown under HL, the spectrally averaged $k_{c}$ of culture 2 rose from 0.0073 to $0.0114 \mathrm{~m}^{2}$ $(\mathrm{mg} \mathrm{chl})^{-1}$, whereas $k_{\mathrm{c}}$ of culture 1 remained constant (Fig. 2). The differences in $k_{\mathrm{c}}$ at $675 \mathrm{~nm}$ indicated that self-shading of thylakoids both within and between the chloroplasts was decreased in culture 2 under HL. This increase in the package effect at lower growth irradiances is well documented (Morel \& Bricaud, 1981; Osborne \& Raven, 1986; Berner et al., 1989). When the $k_{\mathrm{c}}$ of culture 1 is divided by the $k_{c}$ of culture 2 (Fig. 3) changes in pigment composition can be detected. As already discussed above, in LL the ratio relative to that at $675 \mathrm{~nm}$ is higher in the blue to yellow region, indicating that accessory light harvesting pigments were responsible for the slightly higher average cross-section of culture 1 during growth in LL. In contrast to the situation in LL, $k_{\mathrm{c}}$ of culture 2 was uniformly larger than that of culture 1 (Fig. 3), indicating that the pigment composition of both cultures was the same in HL, but that the package effect was more pronounced in culture 1 .

\section{$P_{\max }^{B}$ and $\alpha^{B}$}

Photosynthetic efficiency $\left(\alpha^{\mathrm{B}}\right)$ was not very dependent on the light intensity (Table 1). The small differences between the cultures grown in LL or HL were not significant. However, $\alpha^{\mathrm{B}}$ of $S$. costatum grown under a fluctuating light regime (culture 2) showed higher photosynthetic rates per unit chlorophyll than those from cells grown with the sinusoidal light regime. The maximal photosynthetic capacity in LL was slightly
Table 1. Efficiency of photosynthesis $\left(\alpha^{B}\right)$, photosynthetic capacity $\left(P_{\text {max }}^{B}\right)$ and $I_{k}$ values for cells grown in $L L$ or HL with sinusoidal (culture 1) or fluctuating (culture 2) light climates

The $95 \%$ confidence interval is given between brackets. Culture 1 : Data are means of nine samples taken on three different days, Culture 2: Data are means of 14 samples taken on three different days.

\begin{tabular}{|c|c|c|c|}
\hline Light & $\begin{array}{c}\alpha^{\mathrm{B}} \\
{\left[\mathrm{mg} \mathrm{O}_{2}(\mathrm{mg} \mathrm{chl})^{-1}\right.} \\
\left.\mathrm{h}^{-1}\left(\mu \mathrm{E} \mathrm{m} \mathrm{m}^{-2} \mathrm{~s}^{-1}\right)^{-1}\right]\end{array}$ & $\begin{array}{c}P_{\max }^{\mathrm{B}} \\
{[\mathrm{mg} \mathrm{O}} \\
\left.(\mathrm{mg} \mathrm{chl})^{-1} \mathrm{~h}^{-1}\right]\end{array}$ & $\left(\mu \mathrm{E} \mathrm{m}^{I_{\mathrm{k}}} \mathrm{s}^{-1}\right)$ \\
\hline \multicolumn{4}{|c|}{ Culture 1} \\
\hline LL & $0.091(0.018)$ & $7 \cdot 11(1 \cdot 24)$ & 78 \\
\hline $\mathrm{HL}$ & $0.075(0.015)$ & $9 \cdot 90(2.36)$ & 132 \\
\hline \multicolumn{4}{|c|}{ Culture 2} \\
\hline LL & $0.139(0.019)$ & $5.50(0.82)$ & 40 \\
\hline HL & $0.125(0.019)$ & $15.75(1.73)$ & 126 \\
\hline
\end{tabular}

higher in culture 1 than in culture 2 (Table 1). This is reflected in the lower $I_{\mathrm{k}}$ value of culture 2 compared to that of culture 1 . When the cells were grown with a higher irradiance (HL) $P_{\max }^{\mathrm{B}}$ increased nearly threefold in the culture simulating vertical mixing, whereas the increase in $P_{\max }^{\mathrm{B}}$ in culture 1 was rather limited. $I_{\mathrm{k}}$ values were similar for both cultures in HL. So, despite both cultures receiving identical TDLDs in LL or HL, their photosynthetic parameters differed markedly.

As both cultures had the same dilution rate (i.e. the same growth rate when in steady state), different densities might be expected based on the different photosynthetic characteristics. To check this, we calculated the total amount of oxygen produced per mg chlorophyll $a$ during the $8 \mathrm{~h}$ light period using the values quoted in Table 1 . Photosynthesis was calculated every $5 \mathrm{~min}$ based on the following formulation to describe a photosynthesis light curve: $P^{\mathrm{B}}=P_{\max }^{\mathrm{B}} \tanh \left(I / I_{\mathrm{k}}\right)$ (Jassby \& Platt, 1976). In LL culture 1 produced $1241 \mathrm{mg} \mathrm{O}_{2}$ in the light period, whereas culture 2 produced $1022 \mathrm{mg} \mathrm{O}_{2}$. In $\mathrm{HL}$ this was 1991 and $2310 \mathrm{mg} \mathrm{O}_{2}$, respectively. These are surprisingly small differences considering the different photosynthetic parameters between culture 1 and 2. Apparently both cultures have 'optimized' their photosynthetic performance with regard to their light regime. It also explains why differences in biomass are very small (not shown) between both cultures when grown at the same growth rate.

Oxygen flash yields and overall photosynthetic turnover times

Photosynthetic capacity per unit chlorophyll is a function of the number of PSUs and the turnover time $\tau$ of the photosystems. As shown in Table 2, $\tau$ was similar for 
Table 2. Comparison of PSU size, absorption cross sections $\left(\sigma_{c h}, \sigma_{\text {PSU }}\right)$ and quantum requirements $(\tau)$ of photosynthesis for cells grown in $L L$ or $H L$ with sinusoidal (culture 1) or fuctuating (culture 2) light climates

The SD value is given between brackets. Culture 1: Data are means of six samples taken on two different days. Culture 2: Data are means of 10 samples taken on two different days.

\begin{tabular}{lccccc}
\hline \hline Light & $\begin{array}{c}\text { PSU } \\
\text { size }\end{array}$ & $\begin{array}{c}\sigma_{\mathrm{chl}} \\
\left(\mathrm{nm}^{2}\right)\end{array}$ & $\begin{array}{c}\sigma_{\mathrm{PSU}} \\
\left(\mathrm{nm}^{2}\right)\end{array}$ & $\begin{array}{c}\tau \\
(\mathrm{ms})\end{array}$ & $1 / \Phi_{\mathrm{P}}$ \\
\hline $\begin{array}{l}\text { Culture 1 } \\
\text { LL }\end{array}$ & $1869(274)$ & $0 \cdot 0129$ & $24 \cdot 11$ & $24(6)$ & 11 \\
$\quad$ HL & $1507(225)$ & 0.0129 & $19 \cdot 44$ & $31(3)$ & 13 \\
Culture 2 & & & & & \\
$\quad$ LL & $1161(161)$ & 0.0114 & $13 \cdot 24$ & $31(4)$ & 7 \\
HL & $948(202)$ & $0 \cdot 0169$ & 16.02 & $34(10)$ & 11 \\
\hline \hline
\end{tabular}

both cultures in HL and did not change significantly when the cultures were transferred from LL to HL. Culture 1, however, showed an increase in $\tau$ upon the transfer from LL to HL. Our values for $\tau$ were three to four times higher than those reported for $T$. weisflogii (Post et al., 1985). Both cultures decreased with PSU size when grown at a higher irradiance. A similar decrease in PSU size with increasing growth irradiance was reported for different marine algae (Table 3). The PSU size we found for culture 1 for $S$. costatum was similar to that of T. weisflogii reported by Post et al. (1985). Dubinsky et al. (1986) reported larger PSU sizes for the same strain of $T$. weisflogii, grown under identical conditions as in the studies by Post et al.

A decrease in $\alpha^{\mathrm{B}}$ corresponds to the D-type strategy described by Richardson et al. (1983) (to which they fitted $S$. costatum), and which hypothesizes that changes in light climate are reflected in changes in PSU size $(\alpha$ expressed per cell did not change significantly in culture
2 upon a transfer from LL to HL). We indeed found a decrease in PSU size upon a shift from LL to HL, especially in culture 1 (Table 2). However, not only did the PSU size change, also the number of PSUs decreased from $6.0 \times 10^{5}$ to $4.4 \times 10^{5}$ per cell in culture 1 and from $4.5 \times 10^{5}$ to $2.8 \times 10^{5}$ per cell in culture 2 . So, culture 1 had both larger and more PSUs per cell than culture 2 . However, the cells themselves were also considerably larger in culture 1. Because we made no estimates of cell size, we cannot quantify this and hence were unable to express PSU size and number on a biovolume base.

The light regime had a remarkable influence on PSU size of $S$. costatum: the culture with the fluctuating light regime had a smaller PSU size than the culture with the sinusoidal light regime. Thus, the culture receiving fluctuating light responded by decreasing its PSU size and increasing the number of PSUs per unit chlorophyll.

As the package effect decreased in culture 2 in HL, the optical cross-section of a single chlorophyll molecule increased from $0.0114 \mathrm{~nm}^{2}$ in $\mathrm{LL}$ to $0.0169 \mathrm{~nm}^{2}$ in $\mathrm{HL}$. No change occurred in $\sigma_{\text {chl }}$ in culture 1 between LL and HL. Because $\sigma_{\text {chl }}$ did not change in culture 1, the optical cross-section of a single PSU increased as the number of chlorophyll molecules in the PSU increased (Table 2). It can be seen that an increase in $\sigma_{\mathrm{PSU}}$ is not necessary when the PSU size increases in culture 2. The decrease in PSU size is more than compensated for by the increase in $\sigma_{\text {chl }}$ when the culture is grown in HL, resulting in a net increase from 13.24 to $16.02 \mathrm{~nm}^{2}$ per PSU. These optical cross-sections of the PSUs were similar to those reported for T. weisflogii (Dubinsky et al., 1986).

\section{Minimum quantum requirements}

At low flux densities the probability that a photon hits a reaction centre that is closed by a previous hit is small. Therefore there is a linear relationship between photo-

Table 3. Comparison of PSU size as reported for several species

\begin{tabular}{lccl}
\hline \multicolumn{1}{c}{ Species } & $\begin{array}{c}\text { Irradiance } \\
\left(\mu \mathrm{E} \mathrm{m}^{-2} \mathbf{s}^{-1}\right)\end{array}$ & $\begin{array}{c}\text { PSU } \\
\text { size }\end{array}$ & Reference \\
\hline Skeletonema costatum & 30 & 1869 & This study \\
Thalassiosira weisflogii & 60 & 1507 & This study \\
& 30 & 2890 & Dubinsky et al. (1986) \\
& 70 & 2450 & Dubinsky et al. $(1986)$ \\
& 600 & 2210 & Dubinsky et al. $(1986)$ \\
Isochrysis galbana & 72 & 1840 & Post et al. (1985) \\
& 593 & 2115 & Post et al. (1985) \\
Prorocentrum micans & 30 & 1212 & Dubinsky et al. (1986) \\
Chlorella pyrenoidosa & 70 & 934 & Dubinsky et al. (1986) \\
& 600 & 527 & Dubinsky et al. $(1986)$ \\
& 70 & 1107 & Dubinsky et al. (1986) \\
& 600 & 695 & Dubinsky et al. (1986) \\
& 10 & 2350 & Myers \& Graham (1971) \\
\hline
\end{tabular}



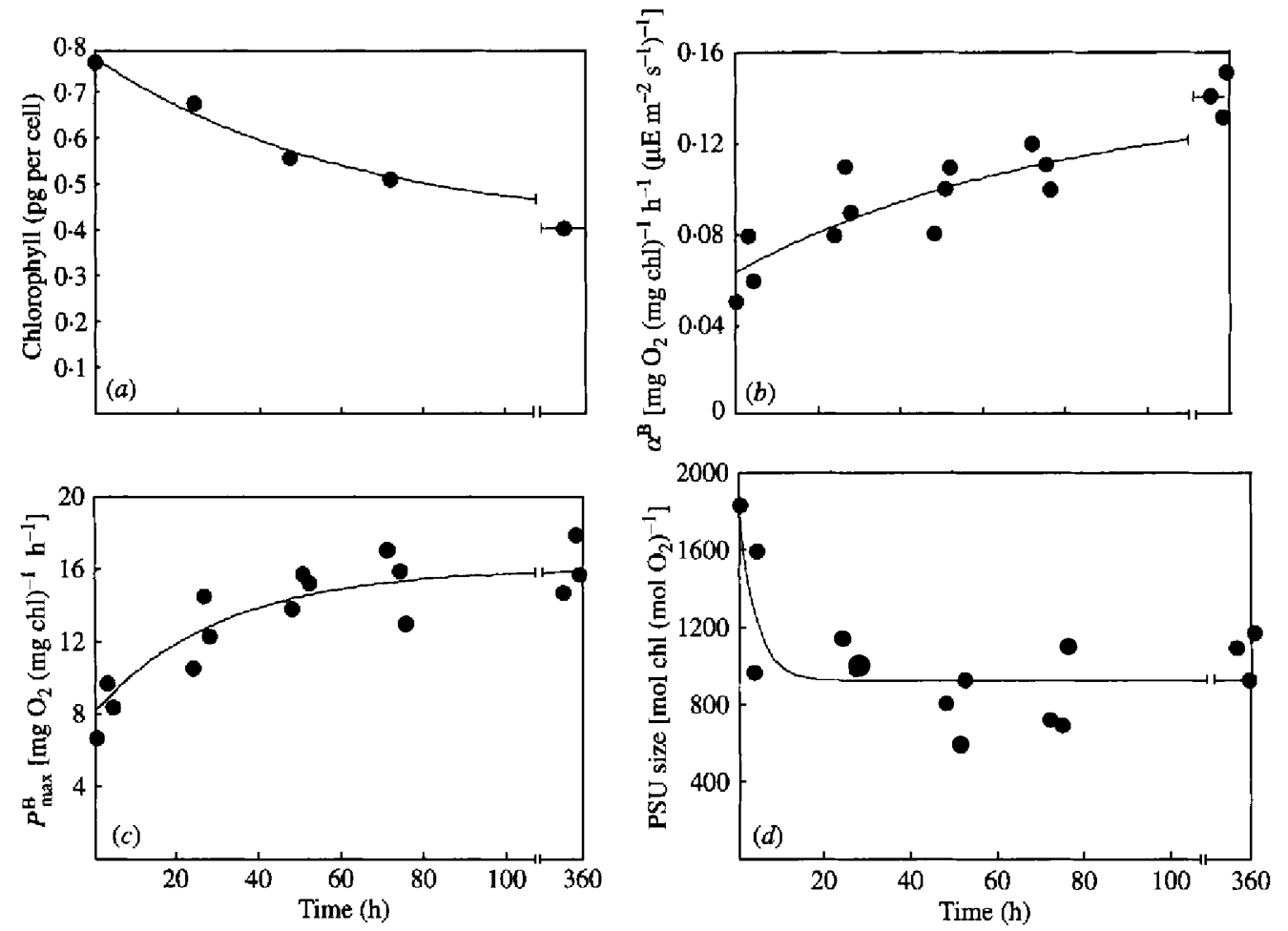

Fig. 4. Change in photosynthetic parameters in culture 2 when at $t=0$ the irradiance was changed from LL to HL. (a) Cellular chlorophyll content; (b) time course of change in photosynthetic efficiency $\left(\alpha^{\mathbf{B}}\right) ;(c)$ time course of change in photosynthetic capacity $\left(P_{\max }^{\mathrm{B}}\right) ;(d)$ time course of change in PSU size.

synthesis and irradiance at low photon flux densities. In this region, up to approximately $I_{\mathrm{k}}$, the quantum requirement will be lowest. We found that the minimum quantum requirements ranged between 7 and 13 , with the higher requirements for the higher irradiances. The value of 7 is actually just below the theoretical minimum of 8 . The slightly higher quantum requirements with increasing growth irradiance might be caused by the increased presence of non-photosynthetically active pigments (Siefermann-Harms, 1985) or by an increase in cyclic photophosphorylation.

\section{Effect of a change in growth irradiance}

The growth irradiance in cultures was increased from an average of 30 (LL) to $60 \mu \mathrm{E} \mathrm{m}^{-2} \mathrm{~s}^{-1}$ (HL) and the photosynthetic parameters followed. As noted above, $\alpha^{B}$ did not change significantly and the changes in $P_{\max }^{\mathrm{B}}$ and $\tau$ were not large enough to allow analyses of the kinetics of the change in values of these two parameters. This was partly because the change in growth irradiance from $\mathrm{LL}$ to $H \mathrm{~L}$ was not very large. Nevertheless, culture 2 showed changes in cellular chlorophyll content, $\alpha^{\mathrm{B}}, P_{\max }^{\mathrm{B}}$ and PSU size (Fig. 4), whereas $\tau$ remained more or less constant. The cellular chlorophyll content decreased following first order kinetics (Fig. 4a). The decrease in pigment content
Table 4. Rate constants describing first order kinetics of photosynthetic parameters of culture $2(L L \rightarrow H L)$

The $r^{2}$ value of the fit is given in parentheses.

\begin{tabular}{cc}
\hline \multicolumn{1}{c}{ Parameter } & $k\left(\mathrm{~h}^{-1}\right)$ \\
\hline $\mathrm{Chl} a(\mathrm{pg})$ per cell & $0.016(0.99)$ \\
$\alpha^{\mathrm{B}}\left[\mathrm{mg} \mathrm{O}_{2}\left(\mathrm{mg} \mathrm{chl}^{-1} \mathrm{~h}^{-1}\left(\mu \mathrm{E} \mathrm{m}^{-2} \mathrm{~s}^{-1}\right)^{-1}\right]\right.$ & $0.013(0.83)$ \\
$P_{\max }^{\mathrm{B}}\left[\mathrm{mg} \mathrm{O}_{2}(\mathrm{mg} \mathrm{chl})^{-1} \mathrm{~h}^{-1}\right]$ & $0.036(0.83)$ \\
PSU $\left[\mathrm{mol} \mathrm{chl} a\left(\mathrm{~mol} \mathrm{O}_{2}\right)^{-1}\right]$ & $0.257(0.56)$ \\
\hline
\end{tabular}

mirrored the increase in $\alpha^{\mathrm{B}}$ (Fig. $4 b$ ). The adaptation rate constants ( $k$ values) were indeed similar for both processes (Table 4). As the specific rate of decrease in the chlorophyll content was similar to the specific rate of increase in cell numbers (not shown), it can be concluded that chlorophyll was diluted by cell division and that it was not actively degraded. The same conclusion was drawn by Post et al. (1984) when $T$. weisflogii was subjected to a large change in irradiance. As $\alpha^{B}$ also changed at the same rate, it seems likely that $k_{\mathrm{c}}$ changed with a similar rate constant. As $\alpha^{\mathrm{B}}=k_{\mathrm{c}}{ }^{*} \Phi_{\mathrm{p}}$ (equation 8), the decrease in the package effect, causing $k_{\mathrm{c}}$ to increase, is to a large extent responsible for the change in $\alpha^{\mathrm{B}} . P_{\max }^{\mathrm{B}}$ increased more rapidly than $\alpha^{\mathrm{B}}$ or the cellular chlorophyll content (Fig. $4 c$ ), but the decrease in PSU size was even 


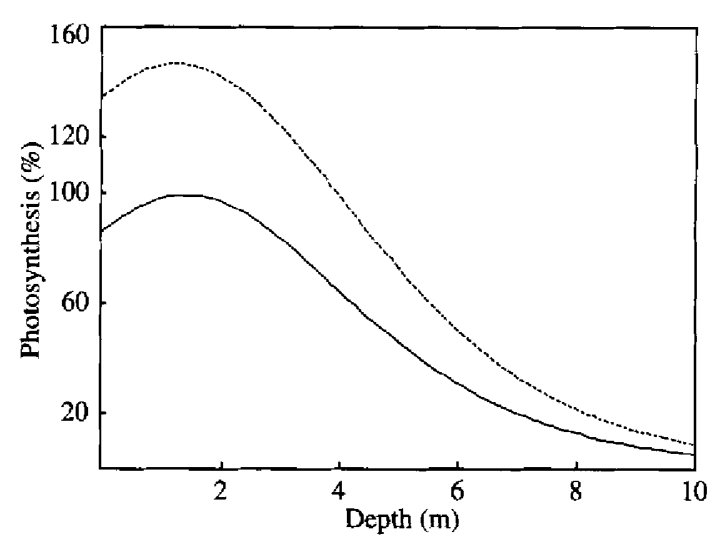

Fig. 5. Theoretical profile of photosynthesis with depth, assuming a downwelling attenuation coefficient of $0.5 \mathrm{~m}^{-1}$. Photosynthesis at different irradiances was calculated according to Eilers \& Peeters (1988; see Methods). For the sample taken at a low culture irradiance $\left(30 \mu \mathrm{E} \mathrm{m}^{-2} \mathrm{~s}^{-1}\right)$ the fit constants $a, b$ and $c$ were $9.1 \times 10^{-5}, 0.066$ and 21 , respectively, whereas for the sample taken at a high irradiance $\left(320 \mu \mathrm{E} \mathrm{m}^{-2} \mathrm{~s}^{-1}\right)$ the constants were $4.4 \times 10^{-5}, 0.058$ and 12 , respectively. The rate was expressed as a percentage of the maximum rate just after the start of the photoperiod when the irradiance in the culture was $10 \mu \mathrm{E} \mathrm{m}^{-2} \mathrm{~s}^{-1}$. Solid line, relative rate of photosynthesis of sample at $10 \mu \mathrm{E} \mathrm{m}^{-2} \mathrm{~s}^{-1}$; dotted line, relative rate of photosynthesis of a sample taken at the first peak in irradiance $\left(320 \mu \mathrm{E} \mathrm{m}^{-2} \mathrm{~s}^{-1}\right)$.

faster. This means that structural changes, i.e. reorganization of pigment complexes took place at a rate higher than the growth rate. As $\tau$ did not change during the transient, changes in $P_{\max }^{\mathrm{B}}$ should be due to changes in PSU size and number (equation 6). However, the change in PSU size was faster than the change in $P_{\max }^{\mathrm{B}}$. A possible explanaion for this might be a redistribution of pigments in the light-harvesting protein complexes of PSI and PSII.

\section{Diel patterns}

We investigated whether both cultures showed any diel pattern in photosynthetic performance. In neither of the steady-state cultures could any change in pigments be detected, hence the $k_{\mathrm{c}}$ values did not change during the day (data not shown). Culture 1 did not reveal any significant diel pattern in photosynthetic activity. Culture 2 (LL), however, showed some variation in photosynthetic properties during the day. The $\alpha^{\mathrm{B}}$ and PSU size did not change significantly during the day, but the turnover time $\tau$, and consequently $P_{\max }^{\mathrm{B}}$, showed some diel activity: when the light intensity in the culture peaked (see Fig. 1), $\tau$ decreased approximately $5 \mathrm{~ms}$, causing an increase in $P_{\max }^{\mathrm{B}}$ (data not shown). Because of this pattern, which was found two out of three times, $P_{\max }^{\mathrm{B}}$ showed lower values at the start and at the end of the light period. When culture 2 was shifted from LL to HL a significant pattern of change in photosynthetic parameters, coinciding with the change in light intensity, was observed during the first $2 \mathrm{~d}$ of the transient state (Fig. 4). Both $P_{\max }^{\mathrm{B}}$ and $\alpha^{\mathrm{B}}$ increased when the culture irradiance increased and decreased again when the irradiance decreased. We calculated the effect of this change in photosynthetic parameters on a theoretical photosynthesis-depth profile (Fig. 5, see legend for details). As can be seen, the sample taken at the start of the photoperiod when the light intensity was still low showed a low primary production compared to the sample taken when the culture irradiance was high (approx. at $t=170 \mathrm{~min}$, see Fig. 1). The latter sample was representative for a sample taken at the surface of a water column, whereas the sample taken at LL at the beginning of the photoperiod was representative for a sample taken at the bottom of the euphotic zone. Consequently, vertical mixing through a light gradient can cause, depending on the rate of mixing, a vertical profile in the mixed layer in photosynthetic parameters. Such profiles have been observed in chlorophyll $/ P_{700}$ ratios in the New York Bight and off the coast of Hawaii (Falkowski, 1983), and in $\alpha^{\mathrm{B}}$ and $P_{\max }^{\mathrm{B}}$ in the Northwest Atlantic Ocean (Platt et al., 1992). The existence of vertical profiles makes it necessary to sample the mixed layer at several depths if an accurate estimate of the total primary production is to be achieved. In the example given (Fig. 5), the water column production of the surface sample is $50 \%$ higher than the sample taken near the bottom of the euphotic zone.

Notice that the observed trend in photosynthetic parameters during the light period is opposite to what might be expected according to light-shade adaptation theory. Platt et al. (1992) also mention changes in $P_{\max }^{8}$ with depth opposite to what might be expected on occasions. However, when our LL and HL cultures were compared, the changes in $\alpha^{\mathrm{B}}$ and $P_{\max }^{\mathrm{B}}$ were as expected and were thus as predicted by light-shade adaptation.

\section{Conclusions}

The results from this preliminary study on the possible effects of vertical mixing on the photosynthetic physiology of the marine diatom $S$. costatum clearly showed that the organism adapted to a fluctuating light climate by decreasing its PSU size and increasing the number of PSUs. This made it possible to profit from the high peak irradiances when it was mixed to the lake surface. This effect was especially pronounced in HL, though the overall quantum dose was still not very high.

When the cultures were transferred from $\mathrm{LL}$ to $\mathrm{HL}$, only the culture in which vertical mixing was simulated showed a threefold increase in $P_{\max }^{\mathrm{B}}$. Hence, neither the total daily light dose, nor the growth rate (as this was kept constant) determined the value of the chlorophyll- 
specific maximal photosynthetic capacity. It is therefore tempting to conclude that in this diatom it is the value of the peak irradiance which determines, at least partly, $P_{\max }^{\mathrm{B}}$. As in both LL and HL the peak intensity was larger than $I_{\mathrm{k}}$ the threshold must be higher. Photosynthesis is fully saturated at values higher than $I_{\mathrm{k}}$, i.e. at $I_{\mathrm{opt}}$. In LL neither cultures ever encountered irradiances higher than $I_{\text {opt }}$, but in HL in culture 2 the peak irradiances nearly equalled $I_{\text {opl }}$. So, we cautiously speculate that $P_{\max }^{\mathrm{B}}$ will change if fully saturating irradiances for photosynthesis are reached, but further research is needed.

\section{References}

Berner, T., Dubinsky, Z. \& Falkowski, P. G. (1989). Photoadaptation and the 'package' effect in Dunaliella tertiolecta (Chlorophyceae). Journal of Phycology 25, 70-78.

Cullen, J. J. \& LeWIS, M. R. (1988). The kinetics of algal photoadaptation in the context of vertical mixing. Journal of Plankton Research 10, 1039-1063.

Denman, K. L. \& Gargett, A. E. (1983). Time and space scales of vertical mixing and advection of phytoplankton in the upper ocean. Limnology and Oceanography 28, 801-815.

Dubinsky, Z., Falkowski, P. G. \& Wyman, K. (1986). Light harvesting and utilization by phytoplankton. Plant \& Cell Physiology 27, 1335-1349.

Dubinsky, Z., Falkowski, P. G., Post, A. F. \& Van Hes, U. M. (1987). A system for measuring phytoplankton photosynthesis in a defined light-field with an oxygen electrode. Joumal of Plankton Research 9, 607-612.

Eilers, P. H. C. \& Peeters, J. C. H. (1988). A model for the relationship between light intensity and the rate of photosynthesis in phytoplankton. Ecological Modelling 42, 199-215.

FAlkowsKı, P. G. (1980). Light-shade adaptation in marine phytoplankton. In Primary Productivity in the Sea, pp. 99-119. Edited by P. G. Falkowski. New York: Plenum Press.

FALKowsKI, P. G. (1983). Light-shade adaptation and vertical mixing of marine phytoplankton: A comparative field study. Journal of Marine Research 41, 215-237.

HarRIS, G. P. (1978). Photosynthesis, productivity and growth: the physiological ecology of phytoplankton. Archiv für Hydrobiologie, Beiheft Ergebnisse der Limnologie 10, 1-171.

HerzIG, R. \& FALKOWSKI, P. G. (1989). Nitrogen limitation in Isochrysis galbana (Haptophyceae). I. Photosynthetic energy conversion and growth efficiencies. Journal of Phycology 25, 462-471.

JASSBY, A. D. \& PLATT, T. (1976). Mathematical formulation of the relationship between photosynthesis and light for phytoplankton. Limnology and Oceanography 21, 540-547.

JEFFREY, S. W. \& HUMPHREY, G. F. (1975). New spectrophotometric equations for determining chlorophylls $a, b, c l$ and $c 2$ in higher plants, algae and natural phytoplankton. Biochemie und Physiologie der Pflanzen 167, 191-194.

Kester, D. R., Duedall, I. W., Connors, N. \& Pytkowtcz, R. M.
(1967). Preparation of artificial sea water. Limnology and Oceanography 12, 176-179.

KIRK, J. T. O. (1983). Light and Photosynthesis in Aquatic Ecosystems. New York: Cambridge University Press.

Kolber, Z., ZeHR, J. \& FAI.KOWSKI, P. (1988). Effects of growth irradiance and nitrogen limitation on photosynthetic energy conversion in photosystem II. Plant Physiology 88, 923-929.

Kromkamp, J., Van den Heuvel, A. \& MuR, L. R. (1989). Phosphorus uptake and photosynthesis by phosphate-limited cultures of Microcystis aeruginosa. British Phycological Journal 24, 347-355.

MarRa, J. (1978). Phytoplankton photosynthetic response to vertical movement in a mixed layer. Marine Biology 46, 203-208.

MOREL, A. \& BRICACD, A. (1981). Theoretical results concerning light absorption in a discrete medium, and its applications to specific absorption of phytoplankton. Deep Sea Research 28A, 1375-1393.

MYERs, J. \& GrahaM, J.-R. (1971). The photosynthetic unit in Chlorella measured by repetitive short flashes. Plant Physiology $\mathbf{4 8}$, 282-286.

Neale, P. J. \& MarRa, J. (1985). Short-term variation of $P_{\max }$ under natural irradiance conditions: a model and its implications. Marine Ecology Progress Series 26, 113-124.

OSBORNE, B. A.\& RAvEN, J. A. (1986). Light absorption by plants and its implications for photosynthesis. Biological Reviews 61, 1-61.

Platt, T., Sathyymdranath, S., Ullhoa, O., Harrison, W. G., HOEPFFNER, N. \& GOES, J. (1992). Nutrient control of phytoplankton photosynthesis in the Western North Atlantic. Nature, London 356, 229-231.

Post, A. F. (1986). Transient state characteristics of adaptation to changes in light conditions for the cyanobacterium Oscillatoria agardhii I. Pigmentation and photosynthesis. Archives of Microbiology 145, 353-357.

Post, A. F., Dubinsky, Z., Wyman, K. \& Falkowski, P. G. (1984). Kinetics of light intensity adaptation in a marine planktonic diatom. Marine Biology 83, 231-238.

Post, A. F., Dubinsky, Z., Wyman, K. \& Falkowski, P. G. (1985). Physiological responses of a marine planktonic diatom to transitions in growth irradiance, Marine Ecology Progress Series 25, 141-149.

Reynolds, C. S., Oliver, R. L. \& Walsby, A. E. (1987). Cyanophyte dominance: the role of buoyancy regulation in dynamic lake environments. New Zealand Journal of Marine and Freshwater Research 21, 379-390.

Richardson, K., Beardall, J. \& Raven, J. A. (1983). Adaptation of unicellular algae to irradiance: an analysis of strategies. New Phytologist 93, 157-191.

RiEgMan, R., Rutgers, M. \& MUR, L. R. (1985). Effects of photoperiodicity and light irradiance on phosphate-limited Oscillatoria agardhii in chemostat cultures. Archives of Microbiology 142, 66-71.

Shibata, K., Benson, A. A. \& Calvin, M. (1954). The absorption spectra of suspensions of living micro-organisms. Biochimica et Biophysica Acta 15, 461-470.

Siffermann-HaRms, D. (1985). Carotenoids in photosynthesis I. Location in photosynthetic membranes and light-harvesting function. Biochimica et Biophysica Acta 811, 325-355.

VAN LIERE, L. \& WALSBY, A. E. (1982). Interactions of cyanobacteria with light. In The Biology of the Cyanobacteria, pp. 9-45. Edited by N. G. Carr \& B. A. Whitton. Oxford: Blackwell Scientific Publications.

Welschmeyer, N. A. \& Lorenzen, C. J. (1981). Chlorophyll-specific photosynthesis and quantum efficiency at subsaturating light intensities. Journal of Phycology 17, 283-293. 